\title{
MULTIPLE MODEL FEEDBACK CONTROL OF SYSTEMS WITH NONSMOOTH NONLINEARITIES
}

\author{
PERUTKA, K[arel]
}

\begin{abstract}
The paper deals with multiple model adaptive control of continuous time system with friction and backlash. The realtime control was realized by $P C$ with technological card and using MATLAB software together with Real-time Toolbox. Real-time Toolbox enables real-time control of laboratory models or setups from MATLAB. There was used several models and methods for identification. The system was "preidentified" using multiple model identification and after that real-time adaptive switching control was used for entire control.
\end{abstract}

Key words: feedback control, MATLAB, multiple model control, nonsmooth nonlinearity

\section{INTRODUCTION}

There are many systems with variable parameters of their model. Moreover, there could be a presence of unmodeled influences. Such systems can be controlled by adaptive control. It is useful to combine the adaptive control with multi-mode system identification.

Nonsmooth nonlinearities are common in practice, in industry. Adaptive control was presented for backlash, deadzone, for systems with actuator failure, with the presence of saturation and with unknown time delay in one book (Tao \& Lewis, 2001).

Nonlinear multiple model control is widely used at discrete systems as well. It was proven that neural networks provide sufficient results and they can be implemented in such control strategy. It is possible to combine the linear adaptive controller with nonlinear adaptive controller based on neural network and taking into account the switching algorithm. It was presented that stability and adequate performance can be obtained together (Fu \& Chai, 2007).

Multiple model control of nonlinear systems can ensure better accordance with the real plant if appropriate switching method is selected.

But it is necessary to guarantee the global asymptotic stability. Multiple identification model can be chosen for different purposes. It is possible to set the multiple model in the way that the identification models have same structure but different initial estimates. After that the controller ensures asymptotic tracking and avoids the bounded identification error if some appears. But it is suggested to realize the stability analysis (Ye, 2008).

Combination of multiple models and adaptive control is still quite new and popular approach. There are two common methods these days, switching, and switching and tuning. If multiple models play more important role at control, the better results of control will be achieved. It is due to fact that adaptive control with one model only gives unsatisfactory results at control of linear systems with large uncertainties. But the idea of multiple models is not young. The first works appear in 1970s. But there was a question how to select appropriate switching strategy (Narendra \& Han, 2011).

There are many applications of multiple model control in practice. For example, the multiple model predictive control was used to control the drainage canal system. There is always some uncertainty in this system caused by hardly predicable rainfall. But the controller design is dependent on precise weather forecast. If some heavy rain is predicted, the amount of water in canal system should be decreased before the rain (van Overloop et al., 2008).

Ball mill coal-pulverizing systems are another example where multiple model control was used. Such systems have strong coupling, therefore it is useful to use decoupling control. If coupling is not so strong decentralized control can be used. It was proven that this approach increase production and decrease the consumption of coal (Chai et al., 2011).

The remainder of the paper is organized as follows. Firstly, the necessary background of the used algorithm is presented. This is followed by the short description of the laboratory setup on which the multiple model control was verified. Finally, the results of control are shown.

\section{MULTIPLE MODEL CONTROL}

The used control method enlarges the method that was introduced and presented by Perutka (Perutka, 2007).

Before the control, the controlled multivariable system is decoupled into a set of single input single output (SISO) systems. Everything is realized in continuous time.

After decoupling, the system is pre-identified by multiple model identification. Two well-known methods of identification were used, namely the least squares and instrumental variable method. One of the results obtained from pre-identification is shown in figure 2 .

It this figure, there are results of pre-identification of first decoupled subsystem obtained by instrumental variable method. The whole time interval of pre-identification was split into intervals acconding to combination of the changes of the reference signals. After that, the obtained results from preidentification were analyzed using correlation coefficient criterion and sorted.

The adaptive control is based on the multiple model preidentification. In each time instant, the new parameters of the model of each subsystem is counted and than the new parameters of continuous-time sub-controller is re-counted. There was used the suboptimal linear quadratic tracking controller which was described by Perutka (Perutka, 2007). But the methods used for on-line identification changes according to the results obtained from pre-identification and their change is controlled by supervisor which realizes the switching procedure.

\section{DESCRIPTION OF LABORATORY SETUP}

The laboratory setup which was used for verification of multiple model control of systems with nonlinearities is shown in figure 1. This laboratory model enables us to solve the control tasks connected with the tension of rewinding material and its speed. Such example can be find in plastic or paper 
industry. There are 3 wheels which are interconnected by flexible belt. Two lower wheels are fixed and directly connected with two bidirectional servomotors. The third wheel is mounted on the jib connected to spring.

The control of speed and extension of flexible belt is the main task of the control. It is realized via two servomotors connected to lower wheels.

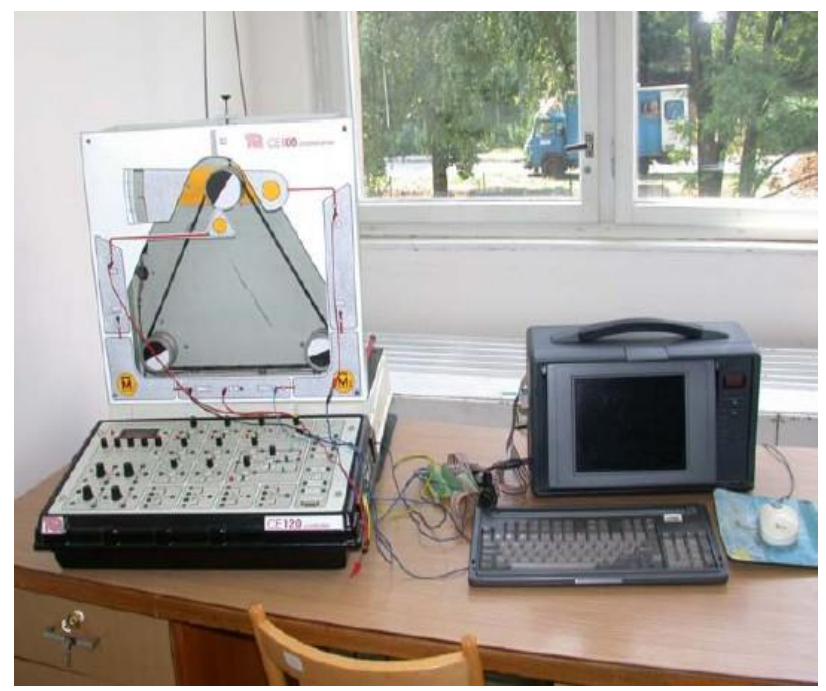

Fig. 1. System of coupled drives connected to PC

\section{RESULTS OF CONTROL}

Figures 2 and 3 shows results connected with real-time multiple model adaptive control of laboratory setup, system with nonlinearities. Figure 2 depicts the selected results of multiple model pre-identification of controlled system. The presented curves are result of instrumental variable method applied on first subsystem of the multivariable model of the controlled system. Curves in this figure show history of parameters of the subsystem model.

Figure 3 shows history of control. There were two subsystems which were controlled by decoupled multiple model control. These systems were marked as subsystem one and two. The meaning of the symbols used in figure 3 is the following one: $w_{1}$ is reference signal of the first decoupled subsystem, $\mathrm{u}_{1}$ is action signal of the first decoupled subsystem and $y_{1}$ is output signal of the first decoupled subsystem. The description is similar for the second subsystem, so $\mathrm{w}_{2}$ is reference signal for the second decoupled subsystem, $\mathrm{u}_{2}$ is action signal of the second decoupled subsystem and $y_{2}$ is output signal of the second decoupled subsystem.

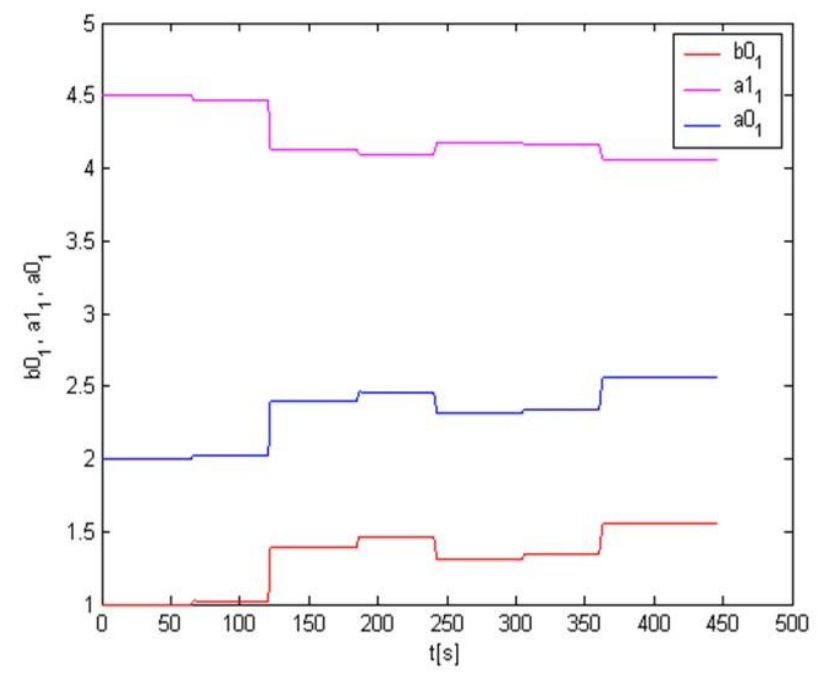

Fig. 2. History of pre-identification of selected model

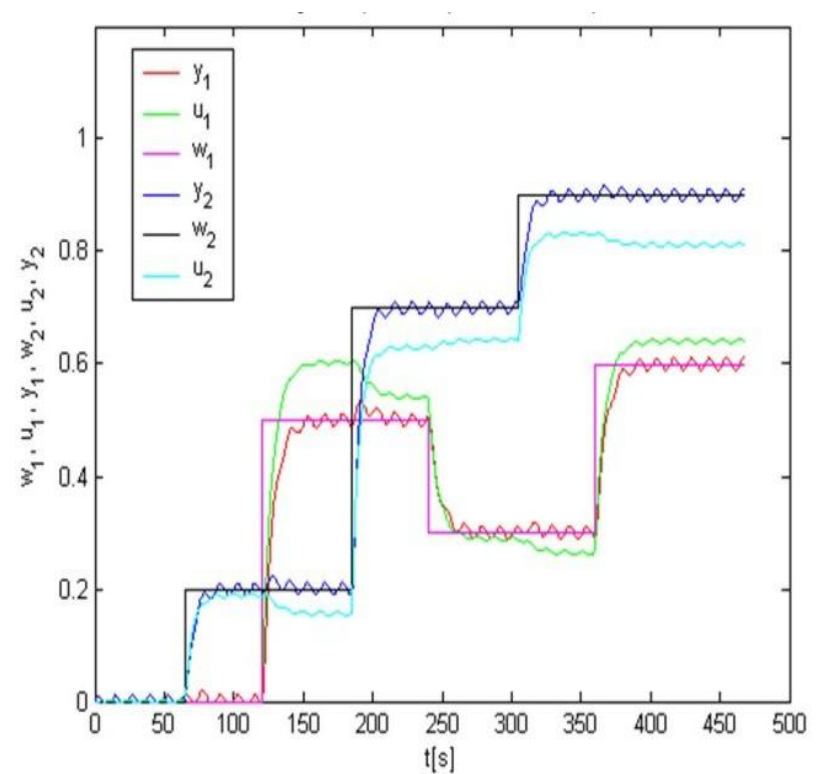

Fig. 3. History of real-time multiple model control of apparatus

\section{CONCLUSION}

The presented results prove that real-time multiple model adaptive decoupled continuous-time control is appropriate method of control for this laboratory setup. The results are satisfactory althought the amount of noise should be decreased.

The control was realized via PC with technological card interconned with the laboratory apparatus. MATLAB and SIMULINK was the software which was used for real-time control.

The future work will take special attention to the reduction of noise for example by implementation of selected filters and to combine the presented approach with robust control to guarantee stability for selected class of systems. It will be also useful to implement another well known method of control and to compare the obtained results.

\section{ACKNOWLEDGEMENTS}

The work was supported by MSM7088352101 grant. The support of this grant is grately acknowledged.

\section{REFERENCES}

Chai, T.; Zhai, L. \& Yue, H. (2011). Multiple models and neural networks based decoupling control of ball mill coalpulverizing systems. Journal of Process Control, Vol. 21, pp. 351-366

Fu, Y., Chai, T. (2007). Nonlinear multivariable adaptive control using multiple models and neural networks. Automatica, Vol. 43, pp. 1101-1110

Narendra, K.S. \& Han, Z. (2011). The changing face of adaptive control: The use of multiple models. Annual Reviews in Control, Vol. 35, pp. 1-12

van Overloop, P.-J., Weijs, S. \& Dijkstra, S. (2008). Multiple Model Predictive Control on a drainage canal system. Control Engineering Practice, Vol. 16, pp. 531-540

Perutka, K. (2007). Decentralized adaptive control, $\mathrm{PhD}$. thesis, Tomas Bata University in Zlin, Zlin. (in Czech)

Tao, G. \& Lewis, L. (2001). Adaptive Control of Nonsmooth Dynamic Systems. Springer-Verlag, ISBN 1-85233-384-7, London, Great Britain

Ye, X. (2008). Nonlinear adaptive control using multiple identification models. Systems \& Control Letters, Vol. 57, pp. $578-584$ 